\title{
Uranium Intermetallics in High Magnetic Fields: Neutron Diffraction Experiments
}

\author{
K. PROKEŠ \\ Hahn Meitner Institute, SF-2, Glienicker Str. 100, 14109 Berlin, Germany \\ The neutron diffraction is an indispensable technique for the determi- \\ nation of magnetic structures. Due to a rather large penetration depth of \\ neutrons in many materials one can construct complicated sample environ- \\ ments necessary to prepare the desired magnetic phase of the material under \\ study. Combination of magnetic fields, low temperatures, and high hydro- \\ static or uniaxial pressures are possible. Capabilities of this technique are \\ demonstrated on a few experimental results obtained on uranium UTX (T: \\ transition metal, $\mathrm{X}$ : p-element) compounds.
}

PACS numbers: $75.25 .+\mathrm{z}$

\section{Introduction}

Uranium-based intermetallics exhibit depending on the delocalization degree of the $5 f$ electron states a large variety of magnetic properties. Systems with a long-range magnetic order, superconductivity, Kondo, heavy-fermion and non-Fermi-liquid states, etc., can be found within a single isostructural group [1]. Due to a specific position of $5 f$ states near the Fermi level, magnetic phenomena are sensitive to external parameters like temperature, magnetic field, and pressure. To understand the magnetic properties of uranium compounds combination of macroscopic and microscopic methods is indispensable. In this contribution, we will present the technique of the neutron diffraction under extreme conditions on few selected examples of quasiternary UTX (T: transition metal, X: $p$-element) compounds. It will be shown that a combined use of external pressure and magnetic field can yield new magnetic phases that can be determined on a microscopic scale with a help of neutron diffraction.

\section{Neutron diffraction and extreme conditions}

Neutron diffraction plays a crucial role in the identification of magnetic structures because the magnetic moment of neutron can interact with periodically arranged magnetic moments resulting to interference phenomena that carry useful 
information regarding the arrangement and size of magnetic moments. The details regarding the theory of the neutron scattering can be found e.g. in [2]. For our purposes it is sufficient to stress that the beam diffracted on periodically arranged atoms or magnetic moments occurs only in specific directions dictated by the Bragg law. This, of course, imposes serious geometrical restrictions for cases when, e.g., the magnetic field is to be used. For experiments two basic experimental geometries exist: with the horizontal or vertical field. The horizontal configuration can be acomplished either with a simple solenoid with a certain opening angle or with a magnet that has a coil split into two or more parts having wedges in the scattering plane. Both designs limit the access for neutrons seriously. In the vertical geometry one has to use a split pair coil design. This has almost no wedges but a smaller maximum field.

From the point of view of the technology to achieve static magnetic fields (the only option for constant-wavelength experiments) one distinguish among superconducting (up to $20 \mathrm{~T}$ ), resistive (up to 30-40 T), and hybrid systems (up to $45 \mathrm{~T}$ ). However, the necessity not to absorb incoming and scattered beams discussed above results in designs offering fields that are much lower. Currently, HMI prepares project to built a $25 \mathrm{~T}$ hybrid solenoid with opening angle of 30 degrees that should be used in conjuction with a dedicated extreme-condition diffractometer [3]. In order to reduce the limitations resulting from rather a small angular range available time of flight method is planned to be used.

Because neutrons interact with most of the elements only weakly, for the diffraction experiments one can use rather complicated sample environment that are required to "prepare" suitable conditions for the existence of particular magnetic phase. This allows us to use cryostats, magnets and to construct suitable pressure cells. The best material for the latter items seems to be Al-based, CuBe and ZrTi alloys with the limiting hydrostatic pressure of about $2.5 \mathrm{GPa}$ and diamond, sapphire or cubic NiBe anvil systems for pressures up to $50 \mathrm{GPa}$, which are combinable with low temperatures and magnetic fields [4].

Neutron diffraction results described in this contribution have been obtained at various institutions, among them at HMI Berlin, LLB Saclay, ILL Grenoble and LANL Los Alamos.

\section{Results}

As the first example we discuss isostructural UTGe $(\mathrm{T}=\mathrm{Co}, \mathrm{Ni}, \mathrm{Rh}, \mathrm{Pd}$ and Ir) compounds crystallizing in the orthorhombic TiNiSi-type of structure (space group Pnma).

UCoGe does not exhibit a long-range magnetic order [1] although it exhibits a strong magnetocrystalline anisotropy documented by a high-field magnetization experiments showing difference for the free to rotate and fixed-powder samples.*

${ }^{*}$ Note added in proof: It has been recently reported by Huy et al. (Phys. Rev. Lett. 99, 067006 (2007) that UCoGe exhibits ferromagnetic order at low temperatures that coexists with superconductivity. 
UNiGe is reported to undergo two magnetic phase transitions at $50 \mathrm{~K}$ and $42.5 \mathrm{~K}$. The ground-state antiferromagnetic (AF) structure is characterized by a propagation vector $(0,1 / 2,1 / 2)$ and ordered moments of about $1.0 \mu_{\mathrm{B}}$. At $4.2 \mathrm{~K}$, there are two field-induced transitions at 17 and $25 \mathrm{~T}$ for the $b$-axis and at 3 and $10 \mathrm{~T}$ for fields along the $c$-axis. No transitions are observed up to $38 \mathrm{~T}$ for the field directed along the $a$-axis. All the magnetic phases, including the field-forced ferromagnetic phase, which is established for the $c$-axis parallel to the applied field above $10 \mathrm{~T}$, have a non-collinear arrangement of $\mathrm{U}$ moments with a significant $a$ axis component. Observation of such a canted ferromagnetic structure provides strong evidence for the presence of anisotropic exchange interactions [5]. Although the latter experiment could be done with the split-pair magnet in the vertical geometry, all other magnetic phases could be studied in the horizontal geometry only. This is because of the existence of the two non-zero propagation vector components.

URhGe orders ferromagnetically $(\mathrm{F})$ below $T_{\mathrm{C}}=9.5 \mathrm{~K}$, and has a lowtemperature ordered uranium moments of $0.4 \mu_{\mathrm{B}}$ oriented along the $c$-axis [6]. The propagation vector of the magnetic structure is thus correspondingly $(0,0,0)$. No $a$-axis AF component has been identified. URhGe exhibits an unconventional $p$-wave superconductivity that apparently coexists with the $\mathrm{F}$ order [6]. One does not expect any influence (except for a change in the moment magnitudes and a rotation of moments towards the field direction) of magnetic field onto the magnetic structure of this compound but on the superconductivity only. Indeed, the superconducting phase is rather quickly suppressed by magnetic field but it recovers at fields around $12 \mathrm{~T}$ if the field is oriented in a narrow angle around the $b$-axis [7]. All the experiments could be done in the vertical geometry.

UPdGe exhibits below $T_{\mathrm{N}}=50 \mathrm{~K}$ AF order with an additional order-order phase transition at $28 \mathrm{~K}$ towards an $\mathrm{F}$ ordering. While the original neutron diffraction studies [8] have claimed that the low-temperature $\mathrm{F}$ state is collinear lying with moments within the $\mathrm{b}-\mathrm{c}$ plane and arbitrary angle with respect to main axes, later studies [9] have shown that the magnetic structure is $\mathrm{F}$ with moments of $1.5 \mu_{\mathrm{B}}$ oriented along the $b$-axis. No $a$-axis component has been found although the high-field magnetization shows a non-negligible zero-field intercept value for this orientation. The absence of metamagnetic transitions is probably also the reason why no detail neutron experiments in high magnetic fields have been performed on this system.

Magnetic measurements on UIrGe single crystal reveal a magnetic phase transition to AF order just above $16 \mathrm{~K}$ and a large magnetic anisotropy with the hard magnetization direction along the $a$ axis [10]. The AF structure of UIrGe is non-collinear and commensurate with the crystallographic unit cell, however, with no $a$-axis component. The four ordered $U$ magnetic moments in the crystallographic unit cell are equal to less than $0.4 \mu_{\mathrm{B}}$ at $1.8 \mathrm{~K}$. For magnetic fields applied along the $b$ - and the $c$-axis, metamagnetic transitions (MT) towards a 
field-induced ferromagnetic phase are observed (14.2 $\mathrm{T}$ at $2 \mathrm{~K}$ for the $c$-axis). Because the propagation vector of the AF structure in UIrGe is $(0,0,0)$, one can use solely the less restrictive vertical geometry.

Clearly, U magnetic moment magnitudes are strongly reduced with respect to $\mathrm{U}^{3+}$ or $\mathrm{U}^{4+}$ single-ion moment values in all the ordered UTGe compounds which is rather common in uranium compounds. One can also follow the development towards the more localized behavior as a consequence of a reduced hybridization between the $5 f$ and ligand states. The ground-state magnetic structure of URhGe and UPdGe is F and collinear, of UIrGe AF with two components and of UNiGe AF with all three components.

The response to the applied magnetic field is highly anisotropic in these compounds. While magnetization measurements in fields lower than $40 \mathrm{~T}$ applied along the hard magnetization axis ( $=a$-axis for all the three compounds) does not indicate any alternation of the magnetic structure, the application of fields of 10-15 $\mathrm{T}$ applied along the $c$-axis induces a field-induced-ferromagnetic (FIF) order in AF ordered compounds. What is interesting is the fact that the FIF state is not collinear for all the compounds although they do show the same type of anisotropy. It seems to be safe to make a conclusion that the $a$-axis component in UNiGe is a consequence of an extremely large anisotropy.

As the second example we choose the case of the hexagonal UNiAl and UNiGa that adopt the ZrNiAl-type of structure. UNiAl crystallizes in the hexagonal ZrNiAl-type structure. In contrast to easy-plane type anisotropy found in the orthorhombic UTX compounds it exhibits a huge uniaxial anisotropy that locks $\mathrm{U}$ magnetic moments along the $c$-axis [1]. The reduced $\mathrm{U}$ moments in UNiAl order below $T_{\mathrm{N}}=19.3 \mathrm{~K}$ in a peculiar AF structure, which is characterized by $\boldsymbol{q}=(0.1,0.1,0.5)[11]$. The metamagnetic transition from the AF ground state to a forced ferromagnetic structure takes place for field applied along the $c$-axis around $11.3 \mathrm{~T}$ at $4.2 \mathrm{~K}$. No transition is seen up to $60 \mathrm{~T}$ with field applied perpendicular to the hexagonal axis. The long-range AF ordering in UNiAl collapses in hydrostatic pressure around $3 \mathrm{GPa}[12]$. A sudden change of the $\rho(T)$ curve observed below $T_{\mathrm{N}}$ for current perpendicular to the $c$-axis at pressures around 0.3 GPa suggested a possibility of a pressure-induced change of the basal plane components of the propagation vector. However, neutron diffraction under hydrostatic pressure up to $0.5 \mathrm{GPa}$ did not reveal any modification of the AF structure. The magnetic phase transition temperature shifts with pressure towards lower values. This is in contrast to the effect of pressure seen in the case of UNiGa, in which it leads to a new pressure-induced phase with a little effect onto the $T_{\mathrm{N}}$ value [13]. The rich magnetic phase diagram is simplified and the only magnetic phase existing in UNiGa at pressures around 2.3 GPa consists of a \pm stacking of ferromagnetic sheets.

The AF structure in UNiAl is, however, modified in an unexpected way by the application of magnetic field directed along the $c$-axis. Before the critical 
field for the metamagnetic-like transition towards the FIF state is reached, the original propagation vector is replaced by another one, namely by $(0.173,0,0.5)$. The magnitude of $U$ moments remain the same for the two structures. Schematic representation is shown in Fig. 1a. In the reciprocal space the magnetic reflections rotate by 30 degrees. The only difference between the zero-field and field-induced AF structure that appears to be stable above $8 \mathrm{~T}$, is the direction of the in-plane modulation. The reason could be found in the details of the Fermi surface.

It is well known that one cannot distinguish between magnetic structures that have several magnetic domains having geometrically equivalent propagation vectors and a monodomain system with a multiple- $q$ magnetic structure. The application of a uniaxial pressure can lift this uncertainty by depopulating one or more of the magnetic domains [3]. Statistical differences in neutron-diffraction data obtained on a single crystal have been interpreted in terms of three magnetic domains [6], however, a direct proof that the AF structure in UNiAl is indeed single- $q$ type have been obtained only recently by applying a small, quasi-uniaxial pressure [14] (see Fig. 1b). An interesting situation occurs if one combines at low temperatures both, the magnetic field and a uniaxial pressure. The field "rotates" the position of magnetic reflections and the pressure depopulates domains in one direction. A natural expectation is that one should be able to produce a monodomain sample as shown in Fig. 1c. Such a combination is a difficult one. The pressure cell has to fit to a magnet that has to be able to generate about $12 \mathrm{~T}$ and to allow for the observation of magnetic reflections that have non-zero $c$-axis component. Such a possibility is achievable only in conjunction with a short wavelength that shifts the magnetic reflections closer to the equatorial plane. The

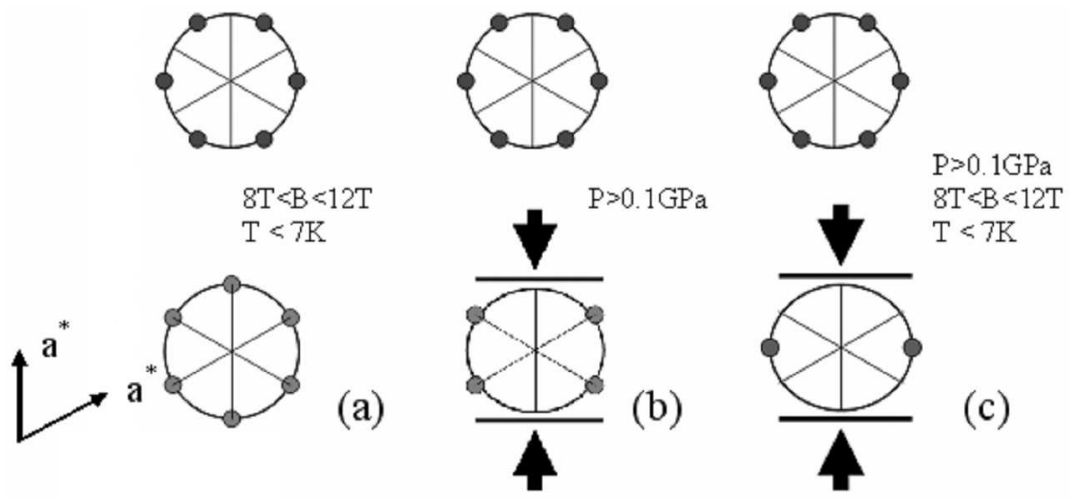

Fig. 1. Schematic representation of the field and pressure effects on the ground state $\mathrm{AF}$ structure in UNiAl in reciprocal space (represented at the top by six reflections). Magnetic field rotates the magnetic reflections by 30 degrees (a). The uniaxial pressure depopulates one of the magnetic domains but leaves the propagation vector unchanged (b). An combined effect of pressure and field produces a monodomain sample (c). 
experiment has been performed recently and shows that, indeed, one can produce a monodomain sample. This is a final proof that the AF structure in UNiAl is not a multiple- $q$ one.

\section{Conclusions}

There is no doubt that the technique of neutron diffraction is indispensable in the solid state research. The necessity to use a various sample environment imposes, however, that sometimes only a very limited set of observables can be measured. Fortunately, the absorption of neutrons for various construction materials is acceptable enabling to construct and combine several extreme conditions at once. However, compromises among the angular range, strength of magnetic field, the value and the type of pressure, the lowest temperature, and the size of studied samples have to be made anyway.

\section{References}

[1] V. Sechovský, L. Havela, in: Handbook of Magnetic Materials, Vol. 11, Ed. K.H.J. Buschow, Elsevier Science B.V., Amsterdam 1998, p. 1.

[2] J. Rossat-Mignot in: Methods of Experimental Physics, Vol. 23, Eds. K.Sköld, D.L. Price, Cambridge Univ. Press, London 1978, p. 389.

[3] J. Peters, K. Lieutenant, D. Clemens, F. Mezei, Z. Kristallogr. Suppl. 23, 189 (2006).

[4] I.N. Goncharenko, High Pressure Research 24, 193 (2004).

[5] K. Prokeš, F. Bourdarot, P. Burlet, P. Javorský, M. Olšovec, V. Sechovský, E. Brück, F.R. de Boer, A.A. Menovsky, Phys. Rev. B 65, 144429 (2002).

[6] D. Aoki, A. Huxley, E. Ressouche, D. Braithwaite, J. Flouquet, J.-P. Brison, E. Lhotel, C. Paulsen, Nature 413, 613 (2001).

[7] F. Levy, I. Sheikin, B. Grenier, A.D. Huxley, Science 309, 1343 (2005).

[8] S. Kawamata, k. Ishimoto, Y. Yamaguchi, T. Komatsubara, J. Magn. Magn. Mater. 104-107, 51 (1992).

[9] S. El-Khatib, S. Chang, H. Nakotte, D. Brown, E. Brück, A.J. Schultz, A. Christianson, A. Acerda,J. Appl. Phys. 93, 8352 (2003).

[10] S. Yoshii, A.V. Andreev, E. Brück, J.C.P. Klaasse, K. Prokeš, F.R. de Boer,. M. Hagiwara, K. Kindo, V. Sechovský, J. Phys., Conf. Series 51, 151 (2006).

[11] K. Prokeš, F. Bourdarot, P. Burlet, P. Javorský, M. Olšovec, V. Sechovský, E. Brück, F.R. de Boer, A.A. Menovsky, Phys. Rev. B 58, 2692 (1998).

[12] K. Prokeš, T. Fujita, N. V. Mushnikov, S. Hane, T. Tomita, T. Goto, V. Sechovsky, A.V. Andreev, A.A. Menovsky, Phys. Rev. B 59, 8720 (1999).

[13] V. Sechovský, K. Prokeš, F. Honda, B. Ouladdiaf, J. Kulda, Appl. Phys. A, Mat. Sci. EJ Proc. 74, S834 (2002).

[14] K. Prokeš, V. Sechovsky, E. Bruck, M. Mihalik, to be published. 